\title{
Survey of Spectra of Laplacians on Finite Symmetric Spaces
}

Audrey Terras

\section{CONTENTS}

1. Introduction

2. Finite Euclidean Symmetric Spaces

3. Finite Non-Euclidean Symmetric Spaces

Acknowledgements

References

Some of the research surveyed here was supported by the Mathematical Sciences Research Institute in Berkeley, CA. Research at MSRI is supported in part by NSF grant DMS-9022140.
We survey what is known about spectra of combinatorial Laplacians (or adjacency operators) of graphs on the simplest finite symmetric spaces. This work is joint with J. Angel, N. Celniker, A. Medrano, P. Myers, S. Poulos, H. Stark, C. Trimble, and $\mathrm{E}$. Velasquez. For each finite field $\mathbb{F}_{q}$ with $q$ odd, we consider graphs associated to finite Euclidean and non-Euclidean symmetric spaces over $\mathbb{F}_{q}$. We are mainly interested in three questions regarding the eigenvalues and eigenfunctions of the combinatorial Laplacian as $q$ goes to infinity: How large is the second largest eigenvalue, in absolute value, compared with the graph's degree? (The largest eigenvalue is the degree.) What can one say about the distribution of eigenvalues? What can one say about the "level curves" of the eigenfunctions?

\section{INTRODUCTION}

In this computer age, it is very natural to model the continuous with the finite: for example, to replace $\mathbb{R}$ with a finite field $\mathbb{F}_{q}$ and the Fourier transform with the fast Fourier transform. Here we begin to do the same thing in higher dimensions.

The goal of this work is to survey what is known about spectra of combinatorial Laplacians, or adjacency operators, for graphs coming from finite analogues of the simplest Euclidean and non-Euclidean symmetric spaces $G / K$ over finite fields of odd characteristic. In a later paper we will consider finite analogues of the Selberg trace formula. In the process we will find some examples of Ramanujan graphs. These graphs have been of interest since the paper of Lubotzky, Phillips and Sarnak [Lubotzky et al. 1988] for their expansion properties, which make them well suited for building communications networks [Friedman 1993]. At the beginning our work was motivated by that of Stark 
[1987] on $p$-adic upper half-planes. We were also inspired by [Arthur 1989; Diaconis 1988; Lubotzky 1994; Sarnak 1990], and by work of Fan Chung and Sternberg on the buckyball [Sternberg 1994]. Graph analogues of Riemann surfaces have been of interest to geometers for some time [Buser 1992].

Much of the work reported on here is joint with Jeff Angel, Nancy Celniker, Archie Medrano, Perla Myers, Steve Poulos, Harold Stark, Cindy Trimble, and Elinor Velasquez. See [Angel et al. 1992; Angel et al. 1994; Angel et al. 1995; Celniker et al. 1993; Medrano et al. a; b; Terras 1991; Stark and Terras]. Section 2 discusses the Euclidean case, mostly following [Medrano et al. a]. Section 3 discusses the non-Euclidean case. See page 28 for a table summarizing this paper.

\section{FINITE EUCLIDEAN SYMMETRIC SPACES}

It is easy to find a finite analogue of $n$-dimensional Euclidean space $\mathbb{R}^{n}$ by replacing $\mathbb{R}$ with the finite field $\mathbb{F}_{q}$ having $q=p^{r}$ elements, where $p$ is an odd prime. We define finite Euclidean $n$-space as the space $\mathbb{F}_{q}^{n}$ of column vectors $x$ with $j$-th entry $x_{j} \in$ $\mathbb{F}_{q}$, for $1 \leq j \leq n$. The distance between two column vectors $x$ and $y$ in $\mathbb{F}_{q}^{n}$ is

$$
d(x, y)={ }^{t}(x-y)(x-y)=\sum_{j=1}^{n}\left(x_{j}-y_{j}\right)^{2},
$$

where ${ }^{t} x$ is the transpose of $x$.

Since it has values in the finite field, this distance is not a metric in the sense of the triangle inequality and analysis, but rather a metric in the sense of quadratic forms and algebra [Lang 1965]. It does possess group invariance properties, just like the Euclidean metric in $\mathbb{R}^{n}$. For clearly

$$
d(x+u, y+u)=d(x, y)
$$

for all $u \in \mathbb{F}_{q}^{n}$, and

$$
d(k x, k y)=d(x, y)
$$

for all $k \in O\left(n, \mathbb{F}_{q}\right)$. Here $O\left(n, \mathbb{F}_{q}\right)$ is the orthogonal group, consisting of $n \times n$ matrices $k$ with entries in $\mathbb{F}_{q}$ such that ${ }^{t} k k=I$, where $I$ is the $n \times n$ identity matrix.

Recall that the Cayley graph of a group $G$ with respect to a set of generators $S$, closed under inversion, has as vertices the elements of $G$ and edges $(g, g s)$, for $s \in S$ and $g \in G$. Since $S$ generates $G$, the graph will be connected; since $S=S^{-1}$, the graph is undirected.

Definition. The Euclidean graph $E_{q}(n, a)$ associated to $a \in \mathbb{F}_{q}$ has as vertices the elements of $\mathbb{F}_{q}^{n}$ and as edges the pairs $(x, y)$ such that $d(x, y)=a$. In other words, it is the Cayley graph of the group $\mathbb{F}_{q}^{n}$ with respect to the set of generators

$$
S_{q}(a)=S_{q}(n, a)=\left\{x \in \mathbb{F}_{q}^{n}: d(x, 0)=a\right\},
$$

except in the exceptional case when $n=2, a=0$, and -1 is not a square in $\mathbb{F}_{q}$; in this case $S_{q}(n, a)=$ $\{0\}$, so that $E_{q}(n, a)$ is then a disconnected graph consisting of $n$ loops. We will usually ignore this exceptional case.

It is not hard to show [Carlitz 1953; Small 1991, pp. 86-91, 145-146] that the graphs $E_{q}(n, a)$, for $a \neq 0$, are regular with degree

$$
\left|S_{q}(n, a)\right|=q^{n-1}+O\left(q^{(n-1) / 2}\right) .
$$

Figure 1 shows two examples.

Definition. The finite Euclidean group $G$ consists of $(n+1) \times(n+1)$ matrices with block form

$$
g=\left(\begin{array}{cc}
k & u \\
0 & 1
\end{array}\right)
$$

where $k \in O\left(n, \mathbb{F}_{q}\right), u$ is a column vector in $\mathbb{F}_{q}^{n}$, and 0 denotes a row vector of $n$ zeros. Then $g$ acts on $x \in \mathbb{F}_{q}^{n}$ by

$$
x \rightarrow k x+u=\left(\begin{array}{cc}
k & u \\
0 & 1
\end{array}\right)\left(\begin{array}{l}
x \\
1
\end{array}\right) .
$$

Note that this action preserves the distance $d(x, y)$ defined in (2.1).

Now define $K$ to be the subgroup of $G$ of matrices with $u=0$. Then $G / K \cong \mathbb{F}_{q}^{n}$. This space is a symmetric space, since we have a commutative algebra 

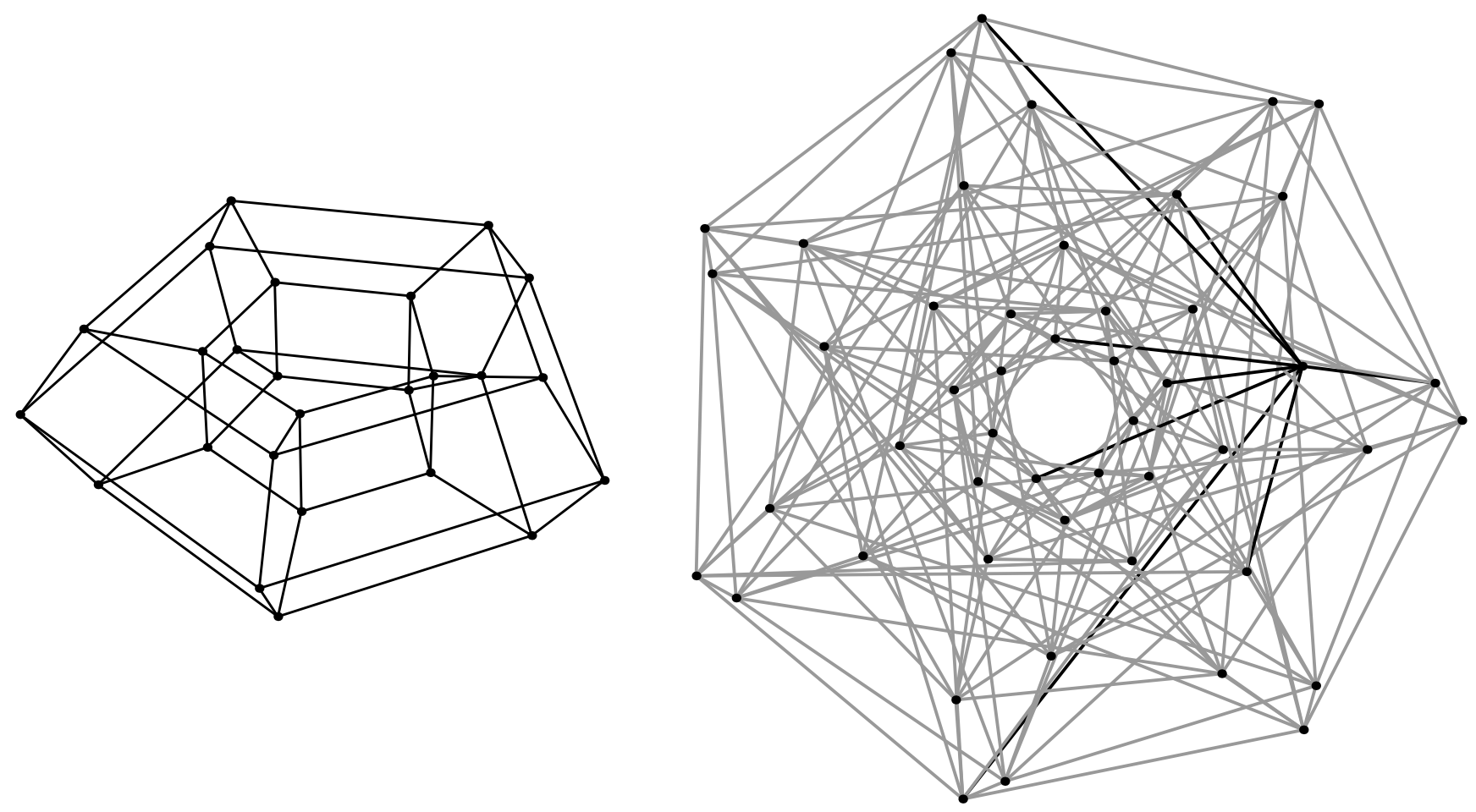

FIGURE 1. Left: The Euclidean graph $E_{5}(2,1)$ has degree four, since $S_{5}(2,1)=\{( \pm 1,0),(0, \pm 1)\}$. Right: $E_{7}(2,1)$ has degree eight, since $S_{7}(2,1)=\{( \pm 1,0),(0, \pm 1),( \pm 2, \pm 2)\}$ (independent signs). The edges incident on one vertex are highlighted.

$L^{2}\left(\mathbb{F}_{q}^{n}\right)$ of functions $f: \mathbb{F}_{q}^{n} \rightarrow \mathbb{C}$ with multiplication defined by convolution:

$$
(f * g)(x)=\sum_{y \in \mathbb{F}_{q}^{n}} f(y) g(x-y) .
$$

As already mentioned, in the exceptional case $(q, n, a)=(q, 2,0)$ with -1 a nonsquare in $\mathbb{F}_{q}$, we have $S_{q}(n, 0)=\{0\}$. Otherwise, we can ask if the $K$-orbits in $\mathbb{F}_{q}^{n}-\{0\}$ are the sets $S_{q}(n, a)$, for $a \neq 0$, or $S_{q}(n, 0)-\{0\}$, for $a=0$. The answer to this question is yes, by Witt's theorem [Lang 1965].

For a symmetric space we need an analogue of the Laplace operator, that is, a $G$-invariant operator on functions on $G / K$.

Definition. The adjacency operator $A=A_{a}$ of a graph $E_{q}(n, a)$ acts on functions $f \in L^{2}\left(\mathbb{F}_{q}^{n}\right)$ by

$$
A_{a} f(x)=\sum_{\substack{y \in \mathbb{F}_{q}^{n} \\ d(x, y)=a}} f(y)=\sum_{u \in S} f(x+u)=\chi_{S} * f(x),
$$

where $d(x, y)$ and $S=S_{q}(n, a)$ are as in (2.1) and (2.2), and $\chi_{S}$ is the function that is 1 on points of $S$ and 0 on points not in $S$. The combinatorial Laplacian is

$$
\Delta=A-d I,
$$

where $d=\left|S_{q}(n, a)\right|$ is the degree of the graph $E_{q}(n, a)$.

This is a self-adjoint, negative operator on $L^{2}\left(\mathbb{F}_{q}^{n}\right)$ with respect to the inner product

$$
\langle f, g\rangle=\sum_{x \in \mathbb{F}_{q}^{n}} f(x) \overline{g(x)},
$$

that is, $\langle\Delta f, g\rangle=\langle f, \Delta g\rangle$ and $\langle\Delta f, f\rangle \leq 0$. This is equivalent to the fact that $A$ is a self-adjoint operator with largest eigenvalue equal to the degree $d$ of the graph. The corresponding eigenfunction is constant. Because the graph is connected if we are not in the exceptional case, the multiplicity of the largest eigenvalue is one [Biggs 1974]. 


\section{Questions One Might Wish Answered}

Question 1. Consider a connected regular graph $X$ of degree $d$ with adjacency operator $A$. Define $\mu=$ $\max \{|\lambda|: \lambda$ is an eigenvalue of $A$ and $\lambda \neq d\}$. We say that $X$ is a Ramanujan graph [Lubotzky et al. 1988] if

$$
\mu \leq 2 \sqrt{d-1}
$$

Are our graphs Ramanujan? This is of interest for various reasons. For example, Ramanujan graphs are good expanders. If $X$ is a connected regular graph, its expansion constant is

$$
c=\max \left\{|\partial Y|: Y \subset X, \text { and }|Y| \leq \frac{1}{2}|X|\right\},
$$

where the boundary $\partial Y$ is the set of $b \in X-Y$ that are adjacent to some vertex in $Y$. The expansion constant $c$ measures transmission speed in a communication network. If $c$ is large, one is happy. The following inequality holds between $\mu, c$, and the degree $d$ of the graph [Sarnak 1990]:

$$
c \geq \frac{1}{2}\left(1-\frac{\mu}{d}\right) \text {. }
$$

One can also show that, for graphs of fixed degree, the Ramanujan graphs are best possible expanders [Lubotzky 1994; Sarnak 1990].

Question 2. What can one say about the distribution of the eigenvalues of $A$-the spectrum? One can ask what happens as $q$ goes to infinity. Do the eigenvalues spread out in a solid line? What about the multiplicities? Look at the histogram of the spectrum (minus the degree). That is, divide up the interval $[-\mu, \mu]$ into subintervals $I$ of equal length and count the number $b(I)$ of eigenvalues in $I$. Then plot rectangles with base I and height $b(I)$. As $q \rightarrow \infty$, does the histogram approach a semicircle? (See (2.8) below.)

Question 3. What can one say regarding the "level curves" of the eigenfunctions?

Question 1 arises in many recent works [Chung 1991; Friedman 1993; Li 1992; Li 1996; Lubotzky 1994; Lubotzky et al. 1988; Sarnak 1990]. Much of the interest in it comes from computer science.
Another reason for interest is that the graph is Ramanujan if and only if the Riemann hypothesis holds for its Ihara zeta function [Hashimoto 1989; Sunada 1988; Venkov and Nitikin 1994; Stark and Terras]. Moreover, there are connections with estimates of classical exponential sums, the Weil conjectures, counting points on curves, the Riemann hypothesis for zeta functions of function fields, as we shall see.

In connection with Question 1, we remark also that good bounds on $\mu$ imply that the simplest random walk on the graph converges extremely rapidly to uniform [Diaconis 1988; Myers 1995].

Question 2 was considered in [Lafferty and Rockmore 1992] for Cayley graphs attached to groups $G$ of $2 \times 2$ matrices with entries in $\mathbb{F}_{q}$ and subsets $S$ of $G$ with $|S|=4$. They found that, as $q \rightarrow \infty$, the spectra approach a line segment with gaps rather than a continuous band. See also [Lafferty and Rockmore 1993].

If the spectra do seem to approach a solid band between $-\mu$ and $\mu$ as $q \rightarrow \infty$, it is natural to ask for even more detailed knowledge. For example, one can ask whether the distribution of the eigenvalues in our problem, like that of a random symmetric matrix, approaches a semicircle as $q \rightarrow \infty$ [McKay 1981; Mehta 1967]. For historical reasons the semicircle distribution is also known as the Wigner semicircle distribution and the Sato-Tate distribution. See formula (2.8) below.

As part of Question 2, one can also ask "what is the level spacing distribution?" [Sarnak a]. This means that you must order the eigenvalues $\lambda_{1} \leq$ $\lambda_{2} \leq \cdots \leq \lambda_{m}$ and then plot the histogram of the differences $\lambda_{i+1}-\lambda_{i}$. The question is whether the histogram looks like the graph of $e^{-x}$.

Question 3 is a finite Euclidean analogue of questions considered in mathematical physics [Courant and Hilbert 1961, p. 302]. See also [Gutzwiller 1990; Hejhal and Rackner 1992; Sarnak 1995].

In the case of eigenfunctions of $\Delta=\partial^{2} / \partial x^{2}+$ $\partial^{2} / \partial y^{2}$ that are zero on the boundary of a domain $D$ in the real plane $\mathbb{R}^{2}$, we are asking for the points of a vibrating drum that reach a given 
height above the plane of the resting drum. By sprinkling dark dust on the membrane one can actually see the zero-level curves, as on the cover of [Powers 1987]. For a circular drum, the contour lines of radial eigenfunctions are circles.

We can think of the simultaneous eigenfunctions $h(x)$, for $x \in \mathbb{F}_{q}^{n}$, of the combinatorial Laplacians on the finite Euclidean graphs $E_{q}(n, a)$ for fixed $q$ and $n$ and varying $a$, as finite spherical functions. We will find that the values of $x$ such that $f(x)$ is constant satisfy $d(x, 0)=$ constant. Thus the level "curves" are finite analogues of circles. See Figure 2 on the next page.

\section{Answers to the Three Questions}

An asymptotic answer to Question 1 is given in [Medrano et al. a]. We summarize the results here. For $y \in \mathbb{F}_{q}$, where $q=p^{r}$ with $p$ prime, define the trace of $y$ as

$$
\operatorname{Tr} y=y+y^{p}+\cdots+y^{p^{r-1}} \in \mathbb{F}_{p} .
$$

Then the exponentials (or characters of the additive group $\mathbb{F}_{q}^{n}$ )

$$
e_{b}(x)=\exp \left(\frac{2 \pi i \operatorname{Tr}\left({ }^{t} x b\right)}{p}\right), \quad \text { for } b, x \in \mathbb{F}_{q}^{n}
$$

are eigenfunctions of the adjacency operator for the Euclidean graphs $E_{q}(n, a)$ corresponding to the eigenvalue

$$
\lambda_{b}=\sum_{d(x, 0)=a} e_{b}(x), \quad \text { for } b \in \mathbb{F}_{q}^{n}
$$

This is an old result and easy to prove: the characters of a finite abelian group are eigenfunctions for the convolution operators on the group.

After some computation, one finds that, when $b \neq 0$, the eigenvalues $\lambda_{b}$ in the formula above are generalized Kloosterman sums, whose definition we recall [Carlitz 1953; Medrano et al. a]. Suppose that $\kappa$ is a character of the multiplicative group
$\mathbb{F}_{q}^{*}$, and let $a, b \in \mathbb{F}_{q}^{*}$. The generalized Kloosterman sum is

$$
K(\kappa \mid a, b)=\sum_{t \in \mathbb{F}_{q}^{*}} \kappa(t) \exp \left(\frac{-2 \pi i}{p} \operatorname{Tr}\left(a t+\frac{b}{t}\right)\right) .
$$

This is a finite analogue of a Bessel function, as can be seen by recalling the formula for the $K$ Bessel function:

$$
K_{s}(y)=\frac{1}{2} \int_{0}^{\infty} t^{s} \exp \left(-\frac{y}{2}\left(t+\frac{1}{t}\right)\right) \frac{d t}{t} .
$$

For this says the $K$-Bessel function is an integral of the product of a multiplicative character of $t$ and an additive character at $t+1 / t$. One can write down a similar formula for the $J$-Bessel function. In the finite case these two functions seem to coincide.

The eigenvalues (other than the degree) of the adjacency operator of $E_{q}(n, a)$ have the form

$$
\lambda_{2 b}=q^{-1} G_{1}^{n} K\left(\varepsilon^{n} \mid a, d(b, 0)\right),
$$

where $G_{1}$ is a Gauss sum defined by

$$
G_{1}=\sum_{t \in \mathbb{F}_{q}} \varepsilon(t) \exp \left(\frac{2 \pi i}{p} \operatorname{Tr} t\right),
$$

and $\varepsilon$ is the quadratic character of $\mathbb{F}_{q}^{*}$, defined by

$$
\varepsilon(t)=\left\{\begin{aligned}
1, & \text { if } t=u^{2} \text { for some } u \in \mathbb{F}_{q}^{*}, \\
0, & \text { if } t=0, \\
-1, & \text { otherwise. }
\end{aligned}\right.
$$

The Gauss sum is a finite analogue of the gamma function, as you can see by recalling Euler's formula

$$
\Gamma(s)=\int_{0}^{\infty} t^{s} \exp (-t) \frac{d t}{t}:
$$

the integral of a multiplicative character times an additive character.

One sees that the eigenvalue $\lambda_{c}$ is also a radial eigenfunction of the combinatorial Laplacian as a function of $c$. This may appear to be rather strange but it is a general phenomenon for symmetric spaces. We will say more about this later. Anyway, once you believe this, it is no surprise that 

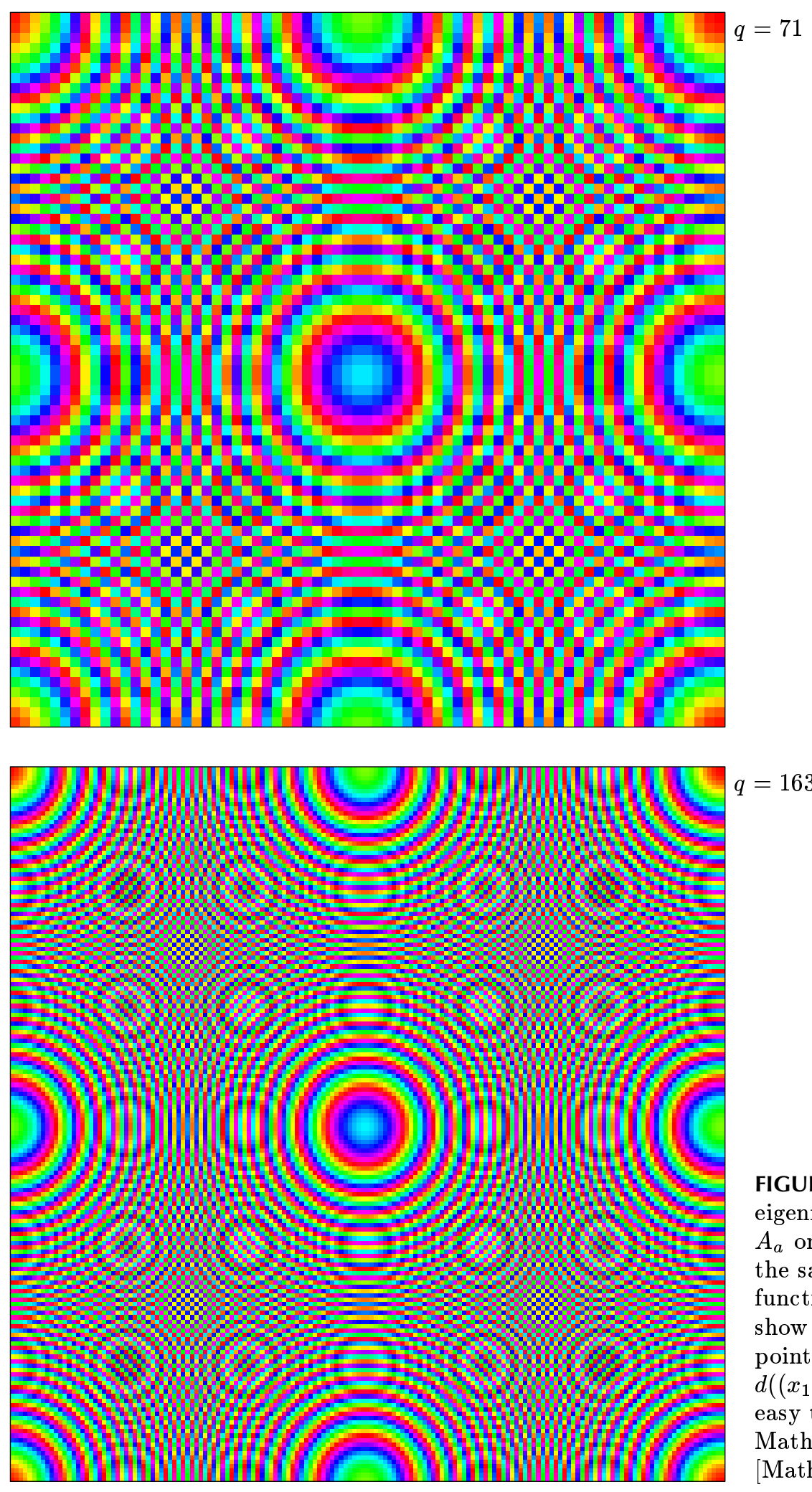

$q=163$

FIGURE 2. A "radial" or $K$-invariant eigenfunction of the adjacency operator $A_{a}$ on a Euclidean graph $E_{q}(n, a)$ has the same level sets as the distance function $d(x, 0)$, for $x \in \mathbb{F}_{q}^{n}$. Here we show a $q \times q$ grid with the color of a point $x$ determined by the distance $d\left(\left(x_{1}, x_{2}\right),(0,0)\right)=x_{1}^{2}+x_{2}^{2} \bmod q$. It is easy to produce such graphs using Mathematica [Wolfram 1992] or Matlab [MathWorks 1995]. 
we have found finite analogues of Bessel functions to be the eigenfunctions of the Laplacian for our Euclidean graphs. See [Terras 1985, Chapter 2].

Weil [1948] bounded the Kloosterman sum (2.4) using his proof of the Riemann hypothesis for zeta functions of curves over finite fields. See [Schmidt 1976] for a more elementary approach, and also [Li 1992, Theorem 16; Li 1996, Theorem 6]. This leads to an asymptotic answer to Question 1 about our Euclidean graphs:

Theorem 2.1 (Finite Euclidean graphs are asymptotically Ramanujan). Let $\lambda_{b}$ be an eigenvalue of the adjacency operator $A$ of the graph $E_{q}(n, a)$ corresponding to the eigenfunction defined by (2.3). Then, for $b \neq 0$, we have the bound $\left|\lambda_{b}\right| \leq 2 q^{(n-1) / 2}$.

The degrees of these graphs are $\left|S_{q}(n, a)\right|=q^{n-1}+$ error. The error may be 0 , positive or negative. If $a \neq 0$ it always is asymptotic to a lower power of $q$ as $q \rightarrow \infty$. This says that the graphs are asymptotically Ramanujan as $q \rightarrow \infty$, in the sense of [Lubotzky et al. 1988]. But one can still ask whether the graphs are exactly Ramanujan. For this, one needs the exact formula for the degree. When $a \neq 0$, it is

$$
\left|S_{q}(n, a)\right|=q^{n-1}+\varepsilon\left((-1)^{(n-1) / 2} a\right) q^{(n-1) / 2}
$$

for $n$ odd, and

$$
\left|S_{q}(n, a)\right|=q^{n-1}-\varepsilon\left((-1)^{n / 2}\right) q^{(n-2) / 2}
$$

for $n$ even. Here $\varepsilon(a)$ is the quadratic character defined in formula (2.5).

For odd $n$, we can see whether our graphs are Ramanujan without too much trouble once we recall a result of Salié [1932] saying that the Kloosterman sums are essentially cosines in this case. A proof can also be found in [Carlitz 1953]. We find that, for odd dimensions $n$,

$$
\begin{gathered}
\lambda_{2 b}=2 G_{1}^{n-1} \varepsilon(d(b, 0)) \cos \left(4 \pi \frac{\operatorname{Tr} c}{p}\right) \\
\text { if } a d(b, 0)=c^{2} \text { with } c \neq 0, \\
\lambda_{2 b}=0 \quad \text { if } a d(b, 0) \text { is not a square } \bmod p,
\end{gathered}
$$

and finally, if $a d(b, 0)=0$ with $b \neq 0$,

$$
\lambda_{2 b}= \begin{cases}q \varepsilon(-a) & \text { if } d(b, 0)=0 \\ q \varepsilon(-d(b, 0)) & \text { if } d(b, 0) \neq 0\end{cases}
$$

From (2.6) and (2.7) it is not hard to see that, if $p \equiv 3 \bmod 4$ and $p>158$, the graphs $E_{p}(3,1)$ are not Ramanujan. Recalling the formula for the degree above, we know that the graphs $E_{p}(3,1)$ for $p \equiv 1 \bmod 4$ are Ramanujan. There can be nonRamanujan graphs $E_{p}(2,1)$ with $p \equiv 1 \bmod 4$ : for example, for $p=17$ and 53 .

It is interesting to note that the graphs $E_{p}(3,1)$ with $p>158$ give examples of similarly constructed graphs whose Ihara zeta functions either do or do not satisfy the Riemann hypothesis according as $p \equiv 1$ or $3 \bmod 4$. We referred to such examples in [Stark and Terras].

It also follows from (2.6) and (2.7) that, for odd dimensions $n$, the eigenvalues can indeed be 0 . In even dimensions, however, [Katz 1980] proves that the Kloosterman sums do not vanish.

Finally, we note that, given $q$ and $n$, there are only two distinct graphs $E_{q}(n, a)$ for even $n$, and at most three for odd $n$ [Medrano et al. a]. This is in contrast to the finite upper half-plane case in the next section, when there seem to be $q-2$ different graphs for each finite field $\mathbb{F}_{q}$.

Before trying to answer Question 2, one should look at Figure 3. The histograms it shows, each for the eigenvalues of a certain Euclidean graph, look very different. The one on the left, for $E_{1021}(2,1)$, looks somewhat like the semicircle distribution one expects for a random graph; the one on the right, for $E_{1019}(3,1)$, does not. In fact, it is proved in [Katz 1988] that the distribution of the Kloosterman sums $K(1 \mid a, 1)$ approaches the semicircle distribution; see also [Adolphson 1989]. More precisely, this means that if $B \subset[-2,2]$ and $\Xi$ is the set of Kloosterman sums $\kappa=K(1 \mid c, 1)$, for $c \in \mathbb{Z} / \mathbb{Z} q$ nonzero, we have

$$
\frac{1}{q-1} \#\left\{\kappa \in \Xi: \frac{\kappa}{\sqrt{q}} \in B\right\} \sim \frac{1}{2 \pi} \int_{x \in B} \sqrt{1-x^{2}} d x
$$



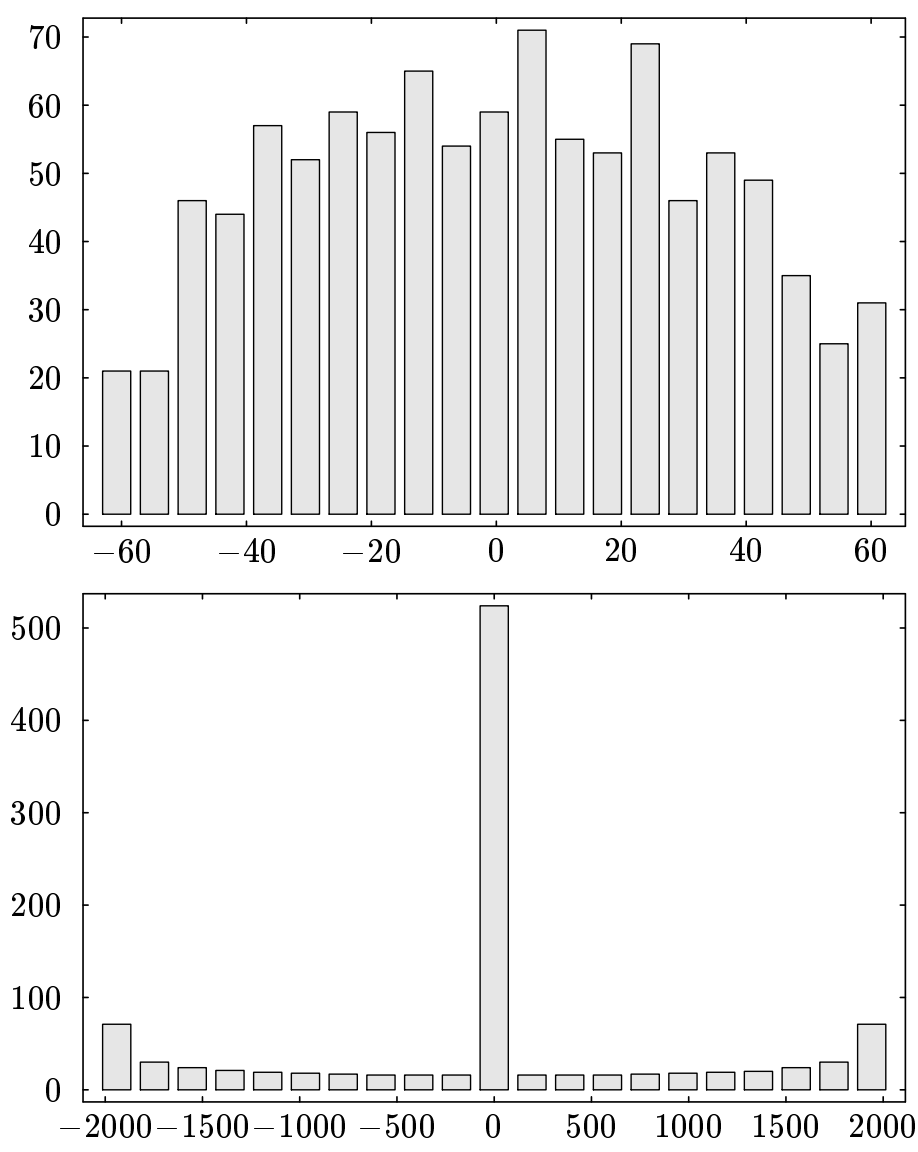

FIGURE 3. Histograms of values of Kloosterman sums for $E_{1021}(2,1)$ (top) and $E_{1019}(3,1)$ (bottom).

as $q \rightarrow \infty$. As in [Adolphson 1989; Katz 1988], take $x=2 \cos \theta$ to obtain the Sato-Tate distribution. See also [McKay 1981; Sarnak 1990]. The moral is that, neglecting the multiplicity of the eigenvalues of $E_{q}(2 n, a)$, their distribution approaches the semicircle distribution as $q \rightarrow \infty$. But in odd dimensions the situation is very different, as Figure 3 shows. Then the eigenvalues are really cosines.

Now we come to Question 3. What are the level "curves" for the eigenfunctions of the adjacency operators for our Euclidean graphs? They are the sets $S_{q}(n, c)$ for fixed $q$ and $n$, as $c$ varies over $\mathbb{F}_{q}$. For $\mathbb{F}_{q}^{2}$, such sets can be viewed as finite analogues of circles, as illustrated in Figure 2, where we associate a color to each element of $\mathbb{F}_{q}$. They look very much like Fresnel diffraction patterns. See [Myers
1995; Goetgheluck 1993] for discussions of similar patterns.

A. Rudvalis suggested that we should look at more general distances than (2.1), such as

$$
d_{c}(x, y)=\sum_{j=1}^{n} c_{j}\left(x_{j}-y_{j}\right)^{2},
$$

where $c=\left(c_{1}, \ldots, c_{j}\right) \in \mathbb{F}_{q}^{n}$. With given $n$ and $q$, one can produce more Ramanujan graphs by varying $c$. Myers [1995] has considered these more general Euclidean graphs. The figures analogous to Figure 2 for $c=(1,-1)$ or $(1, d)$, for some $d \neq 0$ or 1 , are quite interesting: the level "curves" are finite analogues of hyperbolas or ellipses. She also has some beautiful animations of these figures as the prime goes to infinity.

Recently we have also been looking at Euclidean graphs over finite rings $\mathbb{Z} / p^{r} \mathbb{Z}$, and finding that they behave rather differently from those over $\mathbb{F}_{p^{r}}$ in some respects [Medrano et al. b].

\section{FINITE NON-EUCLIDEAN SYMMETRIC SPACES}

If $G$ is a finite group and $K$ is a subgroup, consider the convolution algebra $L^{2}(K \backslash G / K)$ of all $K$ bi-invariant functions $f: G \rightarrow \mathbb{C}$ under the convolution product

$$
(f * g)(x)=\sum_{y \in G} f(y) g\left(x y^{-1}\right) .
$$

If this product is commutative, we call $G / K$ a symmetric space.

In the continuous case, there are many criteria, such as Gelfand's and Selberg's, that ensure that $G / K$ is a symmetric space [Terras 1988; Krieg 1990]. The simplest criterion [Poulos 1991] is just that the $K$-double cosets in $G$ be stable under inversion: $(K s K)^{-1}=K s K$ for all $s \in G$. This is a special case of the Gelfand criterion. The theory of association schemes associated to finite symmetric spaces $G / K$ is also relevant. See [Bannai and Ito 1984] for examples.

Next we want to look at a finite analogue of the Poincaré upper half-plane. The continuous version 
gives a good model for non-Euclidean geometry and has been a symmetric space dear to the hearts of many number theorists [Terras 1985, Chapter 3]. The analogue with $\mathbb{R}$ replaced by a finite field $\mathbb{F}_{q}$ has many charms and has been studied in [Angel 1993; Angel et al. 1992; Angel et al. 1994; Celniker 1994; Celniker et al. 1993; Poulos 1991; Terras 1991]. It is also possible to look at finite analogues over rings $\mathbb{Z} / p^{r} \mathbb{Z}$ [Angel et al. 1995], but these spaces are quite different. For finite fields of even characteristic, see [Angel 1993; Evans 1995].

Let $\mathbb{F}_{q}$ be the field with $q=p^{r}$ elements, where $p$ is an odd prime. Suppose $\delta$ is a generator of the multiplicative group $\mathbb{F}_{q}^{*}$ of nonzero elements in $\mathbb{F}_{q}$. The extension $\mathbb{F}_{q^{2}} \cong \mathbb{F}_{q}(\sqrt{\delta})$ is analogous to $\mathbb{C}=\mathbb{R}[i]$. We define the finite Poincaré upper halfplane as

$$
H_{q}=\left\{z=x+y \sqrt{\delta}: x, y \in \mathbb{F}_{q} \text { and } y \neq 0\right\} .
$$

("Half-plane" is something of a misnomer, since $y \neq 0$ may not be a good finite analogue of the condition $y>0$ that defines the usual Poincaré upper half-plane in $\mathbb{C}$. In fact, $H_{q}$ is more like a double covering of a finite upper half-plane.)

The general linear group $G=\mathrm{GL}\left(2, \mathbb{F}_{q}\right)$ is the group of invertible matrices of the form

$$
g=\left(\begin{array}{ll}
a & b \\
c & d
\end{array}\right)
$$

with $a, b, c, d \in \mathbb{F}_{q}$. The element $\left(\begin{array}{ll}a & b \\ c & d\end{array}\right)$ of $G$ acts on $z \in H_{q}$ by the fractional linear transformation

$$
z \mapsto \frac{a z+b}{c z+d} \in H_{q} .
$$

Moreover, we can easily identify $H_{q}$ with the quotient $G / K$, where $K$ is a finite orthogonal group consisting of matrices $k \in G$ such that

$$
k \sqrt{\delta}=\sqrt{\delta}
$$

It is not hard to see that

$$
K=\left\{\left(\begin{array}{cc}
a & b \delta \\
b & a
\end{array}\right): a, b \in \mathbb{F}_{q} \text { and } a^{2}-b^{2} \delta \neq 0\right\} ;
$$

moreover the correspondence

$$
\left(\begin{array}{cc}
a & b \delta \\
b & a
\end{array}\right) \mapsto a+b \sqrt{\delta}
$$

shows that $K$ is isomorphic to the multiplicative group of $\mathbb{F}_{q}(\sqrt{\delta})$, and hence cyclic.

We use the familiar notation from complex analysis for $z=x+y \sqrt{\delta} \in H_{q}$ :

$$
\begin{aligned}
x & =\operatorname{Re} z, \quad y=\operatorname{Im} z, \\
\bar{z} & =x-y \sqrt{\delta}=z^{q}, \\
N z & =\text { Norm of } z=z \bar{z}=z^{1+q} .
\end{aligned}
$$

The Poincaré distance between $z, w \in H_{q}$ is

$$
d(z, w)=\frac{N(z-w)}{\operatorname{Im} z \operatorname{Im} w} .
$$

As with the Euclidean distance (2.1), this is not a metric in the sense of analysis, but it is $\operatorname{GL}\left(2, \mathbb{F}_{q}\right)$ invariant: $d(g z, g w)=d(z, w)$ for all $g \in \mathrm{GL}\left(2, \mathbb{F}_{q}\right)$ and all $z, w \in H_{q}$.

We can attach graphs to $H_{q}$ by a method analogous to that which led to the Euclidean graphs $E_{q}(n, a)$ in the preceding section.

Definition. For a fixed $a \in \mathbb{F}_{q}$, the finite upper halfplane graph $P_{q}(\delta, a)$ has as vertices the points in $H_{q}$ and edges between vertices $z, w$ if $d(z, w)=a$. Put

$$
\begin{aligned}
R_{q}(\delta, a) & =\left\{z \in H_{q}: d(z, \sqrt{\delta})=a\right\} \\
& =\left\{x+y \sqrt{\delta}: x^{2}=a y+\delta(y-1)^{2}\right\}
\end{aligned}
$$

then $P_{q}(\delta, a)$ is the Cayley graph of the affine group

$$
\left\{\left(\begin{array}{ll}
y & x \\
0 & 1
\end{array}\right): x, y \in \mathbb{F}_{q} \text { and } y \neq 0\right\},
$$

with respect to the set $R_{q}(\delta, a)$, which is invariant under inversion.

Except when $a=0$ or $a=4 \delta, R_{q}(\delta, a)$ is a generating set with $q+1$ elements, so $P_{q}(\delta, a)$ is a connected graph of degree $q+1$. When $a=0$ we have $R_{q}=\{\sqrt{\delta}\}$ and when $a=4 \delta$ we have $R_{q}=\{-\sqrt{\delta}\}$, so that $P_{q}(\delta, a)$ is disconnected in these cases, with one or two nodes, respectively, 
per connected component. As $a$ varies, then, we get $q-2$ graphs $P_{q}(\delta, a)$ of degree $q+1$. It is still an open question whether these graphs are always nonisomorphic.

For example, when $q=3$ you get only one graph: the regular octahedron. For $q=5$ the vertex set corresponds naturally to the vertices of a regular dodecahedron, and the edges of the graph are certain diagonals or edges of the dodecahedron, as shown in Figure 4; there are three possibilities as $a$ varies.

Note also that the sets $R_{q}(\delta, a)$ of (3.2) are the $K$-orbits in $H_{q}$, and can thus be identified with $K$ double cosets in $G=\mathrm{GL}\left(2, \mathbb{F}_{q}\right)$. It follows from all of this that $H_{q}$ is a symmetric space, using the criterion mentioned at the beginning of this section. A representation-theoretic consequence is that any irreducible unitary representation of $G$ occurs in the induced representation $\operatorname{Ind}_{K}^{G} 1$ with multiplicity at most one [Diaconis 1988; Terras 1991].

Remark. Angel [1993] looks at the graphs for finite fields of characteristic two, where one must replace the equation $x^{2}-\delta=0$ with some irreducible quadratic over $\mathbb{F}_{q}$. For example, the graph corresponding to finite upper half-planes over $\mathbb{F}_{4}$ is the icosahedron. See also [Evans 1995]. The references [Angel 1993; Angel et al. 1995] also consider finite upper half-planes over rings like $\mathbb{Z} / \mathbb{Z} p^{r}$. They behave quite differently in some respects.

Just as in Section 2, we define the adjacency operator $A_{a}$ by $A_{a} f=\chi_{R} * f$, where $R=R_{q}(\delta, a)$.

\section{The Same Three Questions}

Question 1. Are the graphs $P_{q}(\delta, a)$ Ramanujan?

Question 2. What can be said about the distribution of the eigenvalues of the adjacency operators of the graphs $P_{q}(\delta, a)$ ?

Question 3. What can be said about the level curves of the eigenfunctions of the adjacency operator of the graphs $P_{q}(\delta, a)$ ?

The answer to Question 1 is yes. For $p$ odd this requires a formula from [Soto-Andrade 1987] and
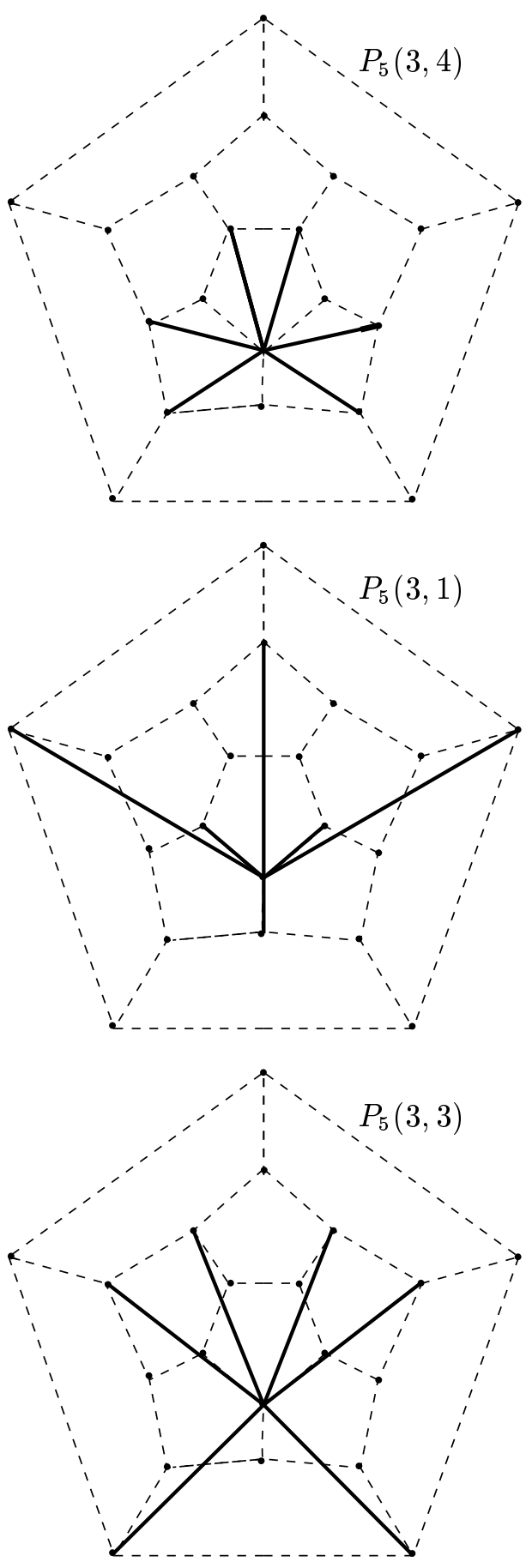

FIGURE 4. We represent the finite upper half-plane $H_{5}$ by a dodecahedron. The edges of the graph $P_{5}(3, a)$ that are incident on a certain vertex are shown as solid lines; the remaining edges can be obtained by applying the dodecahedral symmetries. 
an estimate from [Katz 1993]. Winnie Li [1996; a] shows how to do the estimate without étale cohomology: using $L$-functions of idele class characters of function fields. For finite fields of even characteristic, [Evans 1995] and [Katz 1995] show the graphs are again Ramanujan.

It is possible to derive some understanding of the eigenfunctions of the adjacency operator on $H_{q}$ in a very elementary way. We say a little about that, following [Terras 1985, Chapter 3].

On the continuous Poincaré upper half-plane the power function $p_{s}(z)=(\operatorname{Im} z)^{s}$ is clearly an eigenfunction of the non-Euclidean Laplace operator

$$
\Delta=y^{2}\left(\frac{\partial^{2}}{\partial x^{2}}+\frac{\partial^{2}}{\partial y^{2}}\right)
$$

with eigenvalues $s=s(s-1)$. What is the finite analogue of the power function?

Replace $y^{s}$ with a character $\chi$ of the multiplicative group $\mathbb{F}_{q}^{*}$ of the finite field. Define, for $z \in H_{q}$, the finite power function

$$
p_{\chi}(z)=\chi(\operatorname{Im} z)
$$

Then $p_{\chi}$ is an eigenfunction of the the adjacency operator $A$ of the finite upper half-plane graph $P_{q}(\delta, a)$, with eigenvalue $r_{\chi}$ given by

$$
r_{\chi}=\sum_{z \in R_{q}(\delta, a)} \chi(\operatorname{Im} z)=\sum_{\substack{x, y \in \mathbb{F}_{q}: y \neq 0 \text { and } \\ x^{2}=a y+\delta(y-1)^{2}}} \chi(y) .
$$

There are also analogues of $K$-Bessel functions on the Poincaré upper half-plane $H_{q}$. For the real Poincaré upper half-plane the $K$-Bessel functions are functions $f: H \rightarrow \mathbb{C}$ such that

$$
f(z+u)=\exp (2 \pi i a u) f(z)
$$

for all $u \in \mathbb{R}$ and some $a \in \mathbb{R}$, satisfying the conditions $\Delta f=\lambda f$ and $|f(z)| \leq C y^{p}$. The finite $k$ Bessel function is defined for $\chi$ a multiplicative character of $\mathbb{F}_{q}^{*}$ and $\psi$ an additive character of $\mathbb{F}_{q}$ by

$$
k(z \mid \chi, \psi)=\sum_{u \in \mathbb{F}_{q}} \chi\left(\operatorname{Im} \frac{-1}{z+u}\right) \psi(u) .
$$

This formula is the finite analogue of [Terras 1985, p. 136, eq. (3.14)]. It turns out to be, essentially, a Kloosterman sum [Angel et al. 1992]. One obtains this upon multiplication of $k(z \mid \chi, \psi)$ by a suitable Gauss sum, in analogy with the corresponding continuous result [Terras 1985, p. 136, exercise 1].

The eigenfunction $k(z \mid \chi, \psi)$ turns out to have the same eigenvalue $r_{\chi}$, given in (3.4), as the power function $p_{\chi}(z)=\chi(\operatorname{Im} z)$. Thus the eigenvalues $r_{\chi}$ have multiplicity $\geq p$ for $\chi$ nontrivial. We call them one-dimensional because they actually correspond to one-dimensional representations of the affine group (3.3). There is one more type of eigenvalue, corresponding to a higher-dimensional representation of the affine group. It is easiest to understand this type of eigenvalue by thinking about spherical functions on the symmetric space $G / K$.

In the continuous case of the real Poincaré upper half-plane a spherical function $h: H \rightarrow \mathbb{C}$ is an eigenfunction of $\Delta$ satisfying $h(k z)=h(z)$ for all $k \in K=\mathrm{SO}(2)$ and $z \in H$, normalized by the condition $h(i)=1$. The spherical functions on $H$ are obtained by averaging power functions over $K$ :

$$
h_{s}(z)=\int_{k \in K} p_{s}(k z) d k .
$$

Using polar coordinates $z=k e^{r} i$, where $k \in K$ and $r>0$, we have

$$
h_{s}\left(k e^{r} i\right)=P_{s-1}(\cosh r),
$$

where $P_{\nu}(x)$ is the Legendre function. The level curves of these spherical functions on $H$ are circles.

Recall that $H_{q} \cong G / K$, with $G=\mathrm{GL}\left(2, \mathbb{F}_{q}\right)$ and

$$
K=\left\{\left(\begin{array}{cc}
a & b \delta \\
b & a
\end{array}\right) \in G\right\} .
$$

What are the spherical functions on finite upper half-planes? To find the answer, note that the $G$-invariant operators on $G / K$ are polynomials in the adjacency operators $A_{a}$ of the graphs $P_{q}(\delta, a)$, where $a \in \mathbb{F}_{q}$ (including $a=0,4 \delta$ ). Harmonic analysis on $G / K$ means expanding functions in the common eigenfunctions of the adjacency operators. Moreover, these operators commute, since 
$L^{2}(K \backslash G / K)$ is a commutative algebra under convolution on $G$ (because $G / K$ is a symmetric space); so we can hope to find a simultaneous set of eigenfunctions. In fact, we have already found some: the power function and the Bessel functions. Thus we define a spherical function $h$ on $H_{q}$ as a function $h: H \rightarrow \mathbb{C}$ that is an eigenfunction of all the adjacency operators $A_{a}$ and satisfies $h(k z)=h(z)$ for all $k \in K$ and $z \in H_{q}$, normalized by setting $h(\sqrt{\delta})=1$. We write $h(g \sqrt{\delta})=h(g)$ for $g \in G$.

Remark. There are many references on finite analogues of spherical functions [Angel et al. 1992; Bannai and Ito 1984; Diaconis 1988; Stanton 1990; Terras 1991; Velasquez 1991]. In particular, if $G$ is the semidirect product of $(\mathbb{Z} / n \mathbb{Z})^{k}$ with the symmetric group $S_{k}$ and $K=S_{k}$, one obtains Krawtchouk polynomials as spherical functions. The Krawtchouk polynomials are quite useful in coding theory.

One can also view the spherical functions $h$ as eigenvectors of collapsed adjacency matrices [Angel 1993; Angel et al. 1992; Poulos 1991].

All texts on symmetric spaces, like [Terras 1988], devote a full page to equivalent conditions for a function to be spherical. The most important perhaps is the mean value property.

Mean Value Property. A function $h: H_{q} \rightarrow \mathbb{C}$ is spherical if and only if

$$
\frac{1}{|K|} \sum_{k \in K} h(x k y)=h(x) h(y)
$$

for any $x, y \in G$.

The left-hand side of this equality is $|K|^{-1}\left(A_{a} h\right)(x)$ if $y=y_{a} \in R_{q}(\delta, a)$, the set defined in (3.2). The right-hand side is the eigenfunction $h(x)$ times the eigenvalue $\lambda_{a}=h\left(y_{a}\right)$. The moral of the story is that

\section{eigenfunctions $=$ eigenvalues .}

When you say this to most mathematicians, their hair stands on end. Nevertheless it is true for spherical functions on symmetric spaces.
It turns out that the spherical functions on the symmetric space $G / K$ can be understood by understanding the irreducible unitary representations $\pi$ of $G$ that occur in the regular representation $\rho$ of $G$ on $L^{2}(G / K)$. Because we are dealing with a symmetric space, these representations can occur with at most multiplicity 1 in $\rho$. And it is possible to identify our spherical functions with $K$-fixed vectors $s_{\pi}$ of some irreducible representations $\pi$ of $G$ occurring in $L^{2}(G / K)$. See [Diaconis 1988; Sternberg 1994; Terras 1991] for more details. More explicitly, the spherical function $s_{\pi}$ associated to such an irreducible representation $\pi$ of $G$ is

$$
s_{\pi}(x)=\frac{1}{|K|} \sum_{k \in K} \operatorname{Tr} \pi(k x) .
$$

This can be proved using the Frobenius reciprocity law [Terras 1991].

For our example where $G=\mathrm{GL}\left(2, \mathbb{F}_{q}\right)$ and $K$ is the subgroup fixing $\sqrt{\delta}$, we find two types of spherical functions, and thus, by the preceding equation, two types of eigenvalues, as already mentioned.

The first type, the one-dimensional eigenvalues of formula (3.4), correspond to spherical functions coming from principal series representations of $G$ :

$$
\lambda_{1}(a, b)=\sum_{\substack{x, y \in \mathbb{F}_{q}: y=\delta^{u} \text { and } \\ x^{2}=a y+\delta(y-1)^{2}}} \exp \left(\frac{2 \pi i b u}{q}\right),
$$

where $a, b \in \mathbb{Z} /(q-1) \mathbb{Z}$ with $a \neq 0,4 \delta$.

The second type, corresponding to spherical functions coming from discrete series representations associated to characters $\omega \neq \omega^{q}$ of the multiplicative group $\mathbb{F}_{q}(\sqrt{\delta})^{*}$, were proved in [Soto-Andrade 1987] to have the form

$$
\lambda_{s}(c, \omega)=\sum_{\substack{N z=1 \\ z=x+y \sqrt{\delta}}} \varepsilon(c+2 x) \omega(z),
$$

where $c=(a / \delta)-2$ and $\varepsilon$ is the quadratic character of $\mathbb{F}_{q}^{*}$ defined in (2.5). It is shown in [Soto-Andrade and Vargas 1995] that one can put (3.5) into a form similar to $(3.6)$. 
In order to estimate the eigenvalues, one needs Weil's proof [1948] of the Riemann hypothesis for zeta functions associated to curves over finite fields. Evans and Stark independently used results from [Schmidt 1976] to find estimates for the principal series eigenvalues (3.5), showing that

$$
\left|\lambda_{1}(a, b)\right| \leq 2 \sqrt{q}
$$

for $a \neq 0,4 \delta$ and $1 \leq b \leq q-1$.

N. Katz [1995] used $l$-adic étale cohomology to estimate the Soto-Andrade eigenvalues (3.6). In [Li a] the same estimate is achieved without étale cohomology, using only idele class zeta functions of function fields. The result is

$$
\left|\lambda_{s}(c, \omega)\right| \leq 2 \sqrt{q}
$$

for $1 \leq c \leq q-1, c \neq \pm 2$, and $\omega \neq \omega^{q}$.

Theorem 3.1 (Finite non-Euclidean graphs are Ramanujan). The finite upper half-plane graphs $P_{q}(\delta, a)$, for $a \neq 0,4 \delta$, are Ramanujan graphs.

This completes the discussion of Question 1 for the finite upper half-plane graphs. See also [Evans 1994; Evans 1995].

Next we proceed to Question 2, and ask: Do the eigenvalues have the semicircle (Sato-Tate) distribution? That is, does

$$
\frac{1}{q-1} \#\left\{\lambda: \frac{\lambda}{\sqrt{q}} \in E\right\} \sim \frac{1}{2 \pi} \int_{E} \sqrt{4-x^{2}} d x
$$

as $q \rightarrow \infty$ ? Again we neglect multiplicities, and look only at the $q-1$ eigenvalues given in formulas (3.5) and (3.6). This question is still open. There is some evidence for an affirmative answer. See [Kuang 1995] for a proof that the first and second moments of the eigenvalues asymptotically match those of the semicircle distribution. McKay [1981] shows that, under certain hypotheses, the semicircle distribution is the distribution of the eigenvalues of a large regular graph. But McKay's result assumes that the degree of the graph is fixed and our graphs have degrees going to infinity with $q$.

The histograms in Figure 5 also give some evidence for the conjecture.
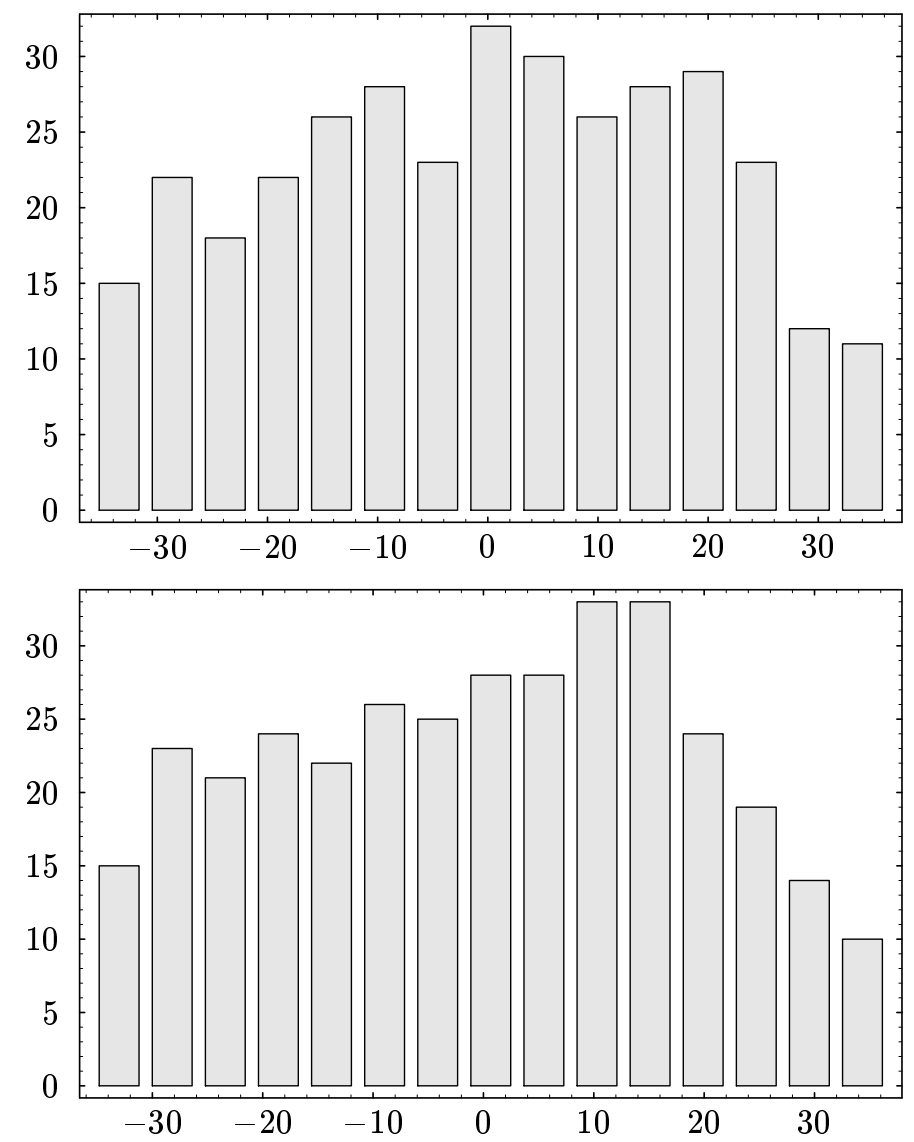

FIGURE 5. Histograms for eigenvalues of $P_{347}(2,2)$ (top) and $P_{347}(2,71)$ (bottom). The height of the bar gives the number of eigenvalues in a subinterval. Data computed by B. Shook using Matlab.

You might ask: What happens if you replace the field $\mathbb{F}_{q}$ by the ring $\mathbb{Z} / q \mathbb{Z}$ with the same number of elements? In this case the graphs fail to be Ramanujan for all $q=p^{2}$ with prime $p>3$, and the eigenvalue distribution looks quite different, as shown in Figure 6. See [Angel et al. 1995].

Finally, we briefly consider Question 3: what can be said about level curves of finite spherical functions $h$ on $H_{q}$ ? For an odd prime $p$, the space $H_{p}$ is a rectangular grid $\left[-\frac{1}{2}(p-1), \frac{1}{2}(p-1)\right] \times[1, p-1]$. We color the square at $(x, j)$ according to the value of the distance

$$
d(x+y \sqrt{\delta}, \sqrt{\delta})=\frac{x^{2}-\delta(y-1)^{2}}{y},
$$



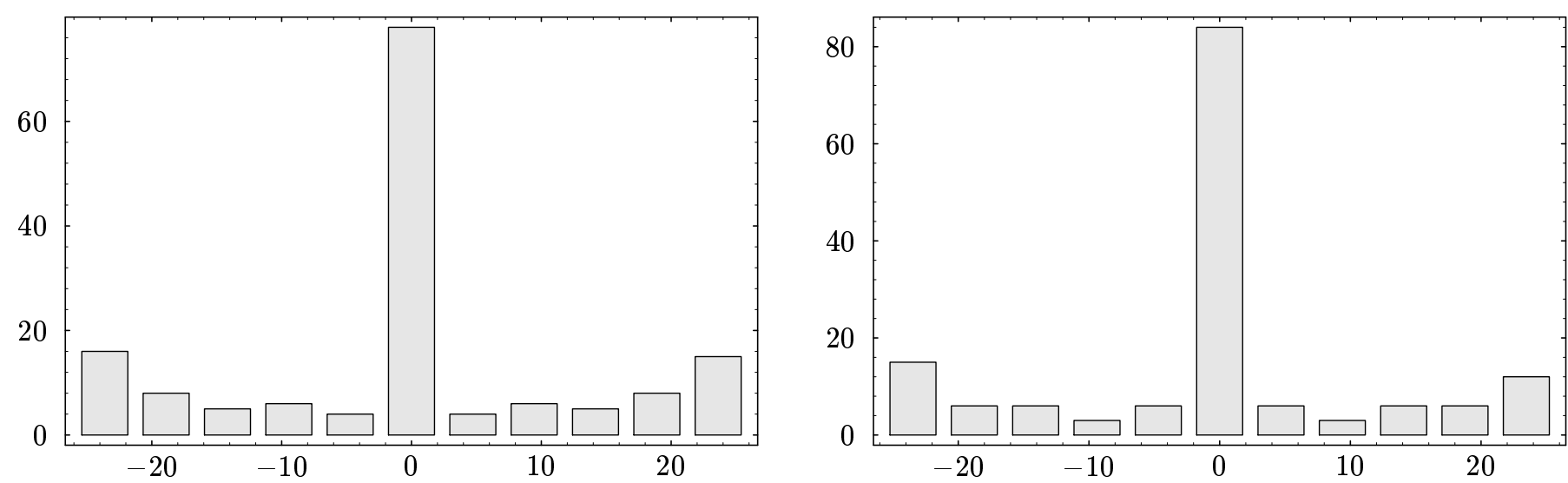

FIGURE 6. Histogram of eigenvalues for finite upper half-planes over the $\operatorname{ring} \mathbb{Z} / 169 \mathbb{Z}$ with $\delta=2$. Left: $a=31$. Right: $a=17$. Data computed by B. Shook using Matlab.

where $y=\delta^{j}$. A level "curve" is obtained by connecting the dots of the same color. The results for $p=71$ and 163 are shown in Figure 7. They look much more chaotic than the analogous figures for the Euclidean graphs Figure 2.

One of the referees asks the interesting questions: Are the non-Euclidean graphs chaotic also from the point of view of "non-Euclidean eyes"? What is the minimum $g$ such that $P_{q}(\delta, a)$ can be drawn on a surface of genus $g$ ? Do the level curves look less chaotic there?

\section{ACKNOWLEDGEMENTS}

I would like to thank R. Evans, N. Katz, A. Rudvalis, B. Shook, and S. Picciotto for their input, and Silvio Levy for editing that goes beyond the call of duty!

\begin{tabular}{|l|l|l|}
\hline & Euclidean & Non-Euclidean \\
\hline space $X \cong G / K$ & $\mathbb{F}_{q}^{n}$ & $H_{q} \subset \mathbb{F}_{q}(\sqrt{\delta})=\mathbb{F}_{q^{2}}$ \\
distance & ${ }^{t}(x-y)(x-y)$ & $\frac{N(z-w)}{\operatorname{Im} z \operatorname{Im} w}$ \\
group $G$ & $\mathbb{F}_{q}^{n} \cdot O\left(n, \mathbb{F}_{q}\right)$ & $\left\{\left(\begin{array}{cc}a & b \delta \\
b & a\end{array}\right) \in G\right\}$ \\
subgroup $K$ & $O\left(n, \mathbb{F}_{q}\right)$ & $P_{q}(n, a)$, for $a \in \mathbb{F}_{q}$ \\
graphs & $E_{q}(n, a)$, for $a \in \mathbb{F}_{q}$ & $q+1$ \\
degree & $q^{n-1}+$ error & $\leq q-2$ \\
$\#$ distinct connected graphs & $\leq 3$ & $R_{q}(\delta, a)=\left\{z \in H_{q}: d(z, \sqrt{\delta})=a\right\}$ \\
connectivity & $S_{q}(a)=\left\{x \in \mathbb{F}_{q}^{n}: d(x, 0)=a\right\}$ & $A_{a} f=\chi_{R_{q}}(\delta, a) * f$ \\
adjacency operator & $A_{a} f=\chi_{S_{q}(a)} * f$ & yes \\
is graph Ramanujan? & asymptotically as $q \rightarrow \infty$ & spherical functions \\
eigenfunctions and eigenvalues & Kloosterman sums & unknown \\
is spectrum a semicircle? & if and only if $n$ is even & unknown (yes if $a=2 \delta)$ \\
is 0 an eigenvalue? & if and only if $n$ is odd & look chaotic \\
level curves & finite Fresnel patterns &
\end{tabular}

TABLE 1. Summary of results: comparison of finite Euclidean and non-Euclidean graphs. 


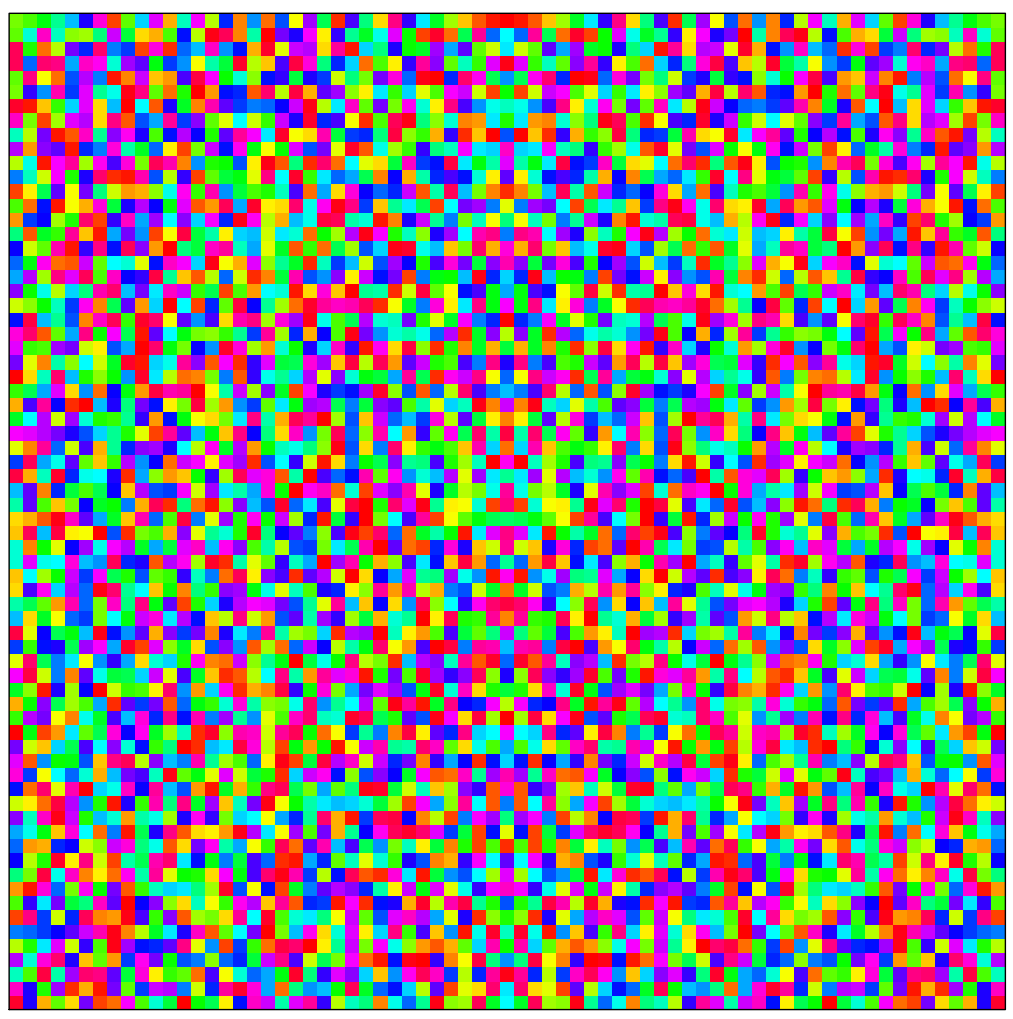

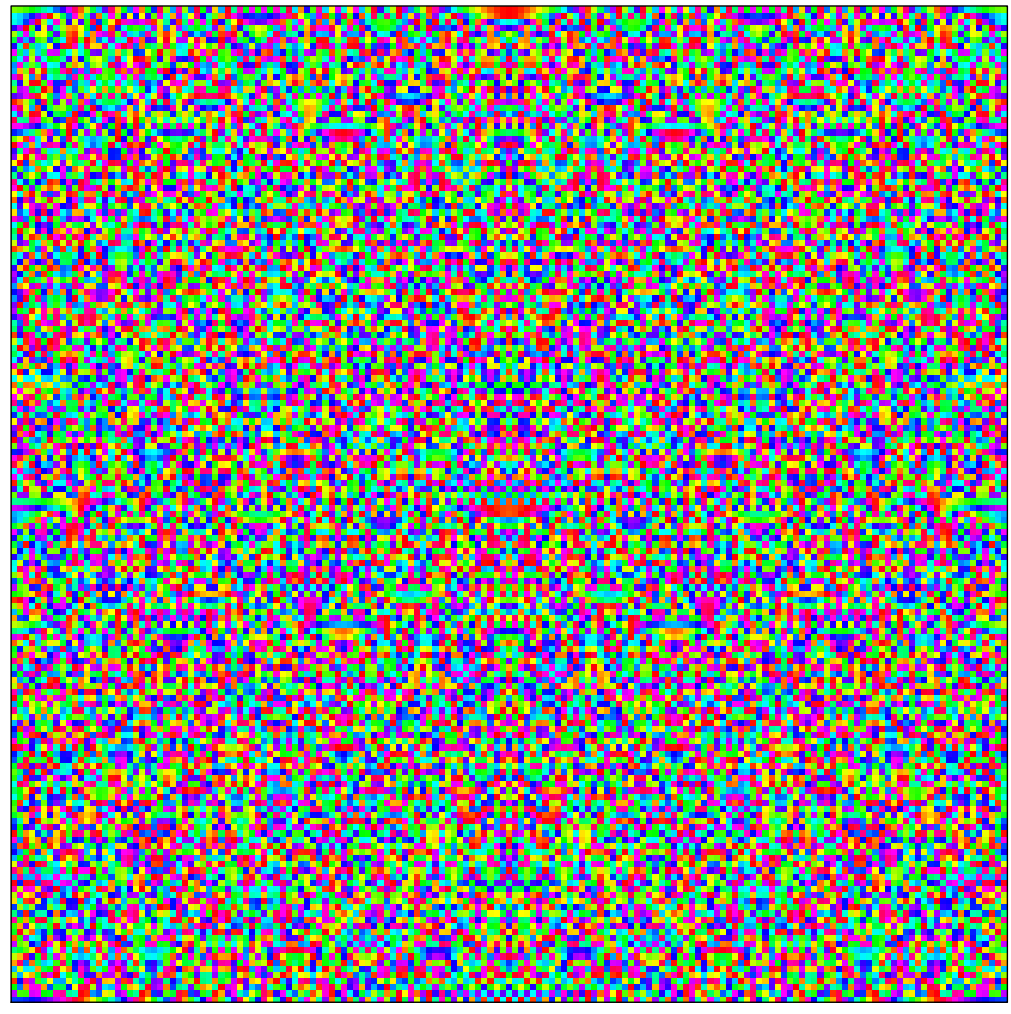

$q=71$

$q=163$ 


\section{REFERENCES}

[Adolphson 1989] A. Adolphson, "On the distribution of angles of Kloosterman sums", J. reine angew. Math. 395 (1989), 214-220.

[Angel 1993] J. Angel, "Finite upper half planes over finite rings and their associated graphs", Ph.D. Thesis, Univ. of California at San Diego, 1993.

[Angel et al. 1992] J. Angel, N. Celniker, S. Poulos, A. Terras, C. Trimble, and E. Velasquez, "Special functions on finite upper half-planes", Contemporary Math. 138 (1992), 1-26.

[Angel et al. 1994] J. Angel, S. Poulos, A. Terras, C. Trimble, and E. Velasquez, "Spherical functions and transforms on finite upper half-planes: eigenvalues of the combinatorial Laplacian, uncertainty, traces", Contemporary Math. 173 (1994), 15-70.

[Angel et al. 1995] J. Angel, B. Shook, A. Terras, and C. Trimble, "Graph spectra for finite upper half-planes over rings", Linear Algebra and its Applications, 226-228 (1995), 423-457.

[Arthur 1989] James Arthur, "The trace formula and Hecke operators", pp. 11-27 in Number Theory, Trace Formulas, and Discrete Groups (edited by K. E. Aubert et al.), Academic Press, Boston, 1989.

[Bannai and Ito 1984] E. Bannai and T. Ito, Algebraic Combinatorics I: Association Schemes, Benjamin/Cummings, Menlo Park, CA, 1984.

[Biggs 1974] N. Biggs, Algebraic Graph Theory, Cambridge U. Press, London, 1974.

[Buser 1992] P. Buser, Geometry and Spectra of Compact Riemann Surfaces, Birkhäuser, Boston, 1992.

[Carlitz 1953] L. Carlitz, "Weighted quadratic partitions over a finite field", Canadian J. Math. 5 (1953), $317-323$.

[Celniker 1994] N. Celniker, "Eigenvalue bounds and girths of graphs of finite, upper half-planes", Pacific J. of Math. 166 (1994), 1-21.

[Celniker et al. 1993] N. Celniker, S. Poulos, A. Terras, C. Trimble and E. Velasquez, "Is there life on finite upper half-planes?", Contemporary Math. 143 (1993), 65-88.

[Chung 1991] F. Chung, Constructing random-like graphs, pp. 21-55 in Probabilistic Combinatorics and Its Applications, San Francisco, 1991 (edited by
B. Bollobás), Proc. Symp. Appl. Math. 44, Amer. Math. Soc., Providence, 1991.

[Courant and Hilbert 1961] R. Courant and D. Hilbert, Methods of Mathematical Physics I, Wiley, New York, 1961.

[Diaconis 1988] P. Diaconis, Group Representations in Probability and Statistics, Lecture Notes-Monograph Series 11, Inst. Math. Statistics, Hayward, CA, 1988.

[Evans 1995] R. Evans, "Spherical functions for finite upper half-planes with characteristic two", Finite Fields and Their Applications 1 (1995), 376-394.

[Evans 1994] R. Evans, "Character sums as orthogonal eigenfunctions of adjacency operators for Cayley graphs", Contemporary Math. 168 (1994), 33-50.

[Friedman 1993] J. Friedman (editor), Expanding Graphs, Princeton, 1992, DIMACS Series in Discrete Math. and Theor. Comp. Sci. 10, Amer. Math. Soc., Providence, 1993.

[Goetgheluck 1993] P. Goetgheluck, "Fresnel zones on the screen", Experimental Math. 2 (1993), 301-309.

[Gutzwiller 1990] M. Gutzwiller, Chaos in Classical and Quantum Mechanics, Springer, New York, 1990.

[Hashimoto 1989] K. Hashimoto, "Zeta functions of finite graphs and representations of $p$-adic groups", pp. 211-280 in Automorphic Forms and Geometry of Arithmetic Varieties (edited by K. Hashimoto and Y. Namikawa), Advanced Studies in Pure Math. 15, Academic Press, Boston, 1989.

[Hejhal and Rackner 1992] D. Hejhal and B. Rackner, "On the topography of Maass waveforms for $\operatorname{PSL}(2, \mathbb{Z})$ ", Experimental Math., 1 (1992), 275-305.

[Katz 1980] N. Katz, Sommes Exponentielles, Astérisque 79 (1980), Soc. Math. France, Paris, 1-209.

[Katz 1988] N. Katz, Gauss Sums, Kloosterman Sums, and Monodromy Groups, Princeton U. Press, Princeton, 1988.

[Katz 1993] N. Katz, "Estimates for Soto-Andrade sums", J. reine angew. Math. 438 (1993), 143-161.

[Katz 1995] N. Katz, "A note on exponential sums", Finite Fields Appl. 1 (1995), 395-398.

[Krieg 1990] A. Krieg, Hecke Algebras, Memoirs Amer. Math. Soc. 87 (\#435) (1990), 158 pp.

[Kuang 1995] J. Kuang, "Eigenfunctions on the finite Poincaré plane", preprint available at http://www. msri.org/MSRI-preprints/online/1995-015.html. 
[Lafferty and Rockmore 1992] J. D. Lafferty and D. Rockmore, "Fast Fourier analysis for $\mathrm{SL}_{2}$ over a finite field and related numerical experiments", Experimental Math. 1 (1992), 115-139.

[Lafferty and Rockmore 1993] J. D. Lafferty and D. Rockmore, "Numerical investigation of the spectrum for certain families of Cayley graphs", pp. 63-73 in [Friedman 1993].

[Lang 1965] S. Lang, Algebra, Addison-Wesley, Reading, MA, 1965.

[Li 1992] W.-C. W. Li, "Character sums and Abelian Ramanujan graphs", J. Number Th. 41 (1992), 199217.

[Li 1996] W.-C. W. Li, "A survey of Ramanujan graphs", in Arithmetic, Geometry, and Coding Theory, Luminy, France, 1993 (edited by R. Pellikaan et al.), de Gruyter, Berlin, 1996.

[Li a] W.-C. W. Li, "Some character sum estimates", preprint.

[Lubotzky 1994] A. Lubotzky, Discrete Groups, Expanding Graphs, and Invariant Measures, Birkhäuser, Basel, 1994.

[Lubotzky et al. 1988] A. Lubotzky, R. Phillips, and P. Sarnak, "Ramanujan graphs", Combinatorica, 8 (1988), 261-277.

[McKay 1981] B. D. McKay, "The expected eigenvalue distribution of a large regular graph", Linear Algebra Appl. 40 (1981), 203-216.

[MathWorks 1995] A. Lubotzky, The MathWorks Inc., MATLAB Version 4 User's Guide, Prentice Hall, Englewood Cliffs, NJ, 1995.

[Medrano et al. a] A. Medrano, P. Myers, H. Stark, and A. Terras, "Finite analogues of Euclidean space", to appear in J. Comp. Appl. Math. 68 (1996).

[Medrano et al. b] A. Medrano, P. Myers, H. Stark, and A. Terras, "Finite Euclidean graphs over rings", preprint.

[Mehta 1967] M. L. Mehta, Random Matrices and the Statistical Theory of Energy Levels, Academic Press, New York, 1967.

[Myers 1995] P. Myers, "Euclidean and Heisenberg graphs: spectral properties and applications", Ph.D. Thesis, Univ. California at San Diego, 1995.
[Poulos 1991] S. Poulos, "Graph theoretic and spectral properties of finite upper half-planes", Ph.D. Thesis, Univ. California at San Diego, 1991.

[Powers 1987] D. Powers, Boundary Value Problems, 3rd ed., Harcourt, Brace, Jovanovich, San Diego, 1987.

[Salié 1932] H. Salié, Über die Kloostermanschen Summen $S(u, v ; q)$, Math. Z. 34 (1932), 91-109.

[Sarnak 1990] P. Sarnak, Some Applications of Modular Forms, Cambridge U. Press, Cambridge, 1990.

[Sarnak 1995] P. Sarnak, "Arithmetic quantum chaos", pp. 183-236 in The Schur Lectures (Tel Aviv, 1992), Israel Math. Soc. Conf. Proc. 8, Bar-Ilan University, Ramat-Gan, 1995.

[Sarnak a] P. Sarnak, "Spectra of hyperbolic surfaces", preprint, Princeton University, 1995.

[Schmidt 1976] W. Schmidt, Equations over Finite Fields: An Elementary Approach, Lecture Notes in Math. 536, Springer, New York, 1976.

[Small 1991] C. Small, Arithmetic of Finite Fields, Dekker, New York, 1991.

[Soto-Andrade 1987] J. Soto-Andrade, "Geometrical Gel'fand models, tensor quotients, and Weil representations", pp. 305-316 in Arcata Conference on Representations of Finite Groups (Arcata, CA, 1986), Proc. Symp. Pure Math. 47, part 2, Amer. Math. Soc., Providence, 1987.

[Soto-Andrade and Vargas 1995] J. Soto-Andrade and J. Vargas, "Harmonic analysis on the twisted finite Poincaré upper half plane", preprint available at http://www.msri.org/MSRI-preprints/online/1995073.html.

[Stanton 1990] D. Stanton, "An introduction to group representations and orthogonal polynomials", pp. 419-433 in Orthogonal Polynomials: Theory and Practice (edited by P. Nevai and M. E. H. Ismail), Kluwer, Dordrecht, 1990.

[Stark 1987] H. Stark, "Modular forms and related objects", Canadian Math. Soc. Conf. Proc. 7 (1987), 421-455.

[Stark and Terras] H. Stark and A. Terras, "Zeta functions of finite graphs and coverings", to appear in Advances in Math.

[Sternberg 1994] S. Sternberg, Group Theory and Physics, Cambridge U. Press, Cambridge, 1994. 
[Sunada 1988] T. Sunada, "Fundamental groups and Laplacians", pp. 248-277 in Geometry and Analysis on Manifolds, Katata/Kyoto, 1987 (edited by T. Sunada), Lecture Notes in Math. 1339, Springer, New York, 1988.

[Terras 1985] A. Terras, Harmonic Analysis on Symmetric Spaces and Applications I, Springer, New York, 1985.

[Terras 1988] A. Terras, Harmonic Analysis on Symmetric Spaces and Applications II, Springer, New York, 1988.

[Terras 1991] A. Terras, "Fourier analysis on finite groups and applications", Lecture Notes, Univ. California San Diego, 1991-1992.
[Velasquez 1991] , E. Velasquez, "The Radon transform on finite groups", Ph.D. Thesis, Univ. California San Diego, 1991.

[Venkov and Nitikin 1994] A. B. Venkov and A. M. Nikitin, "The Selberg trace formula, Ramanujan graphs, and some problems of mathematical physics", Petersburg Math. J. 5 (1994), 419-484.

[Weil 1948] A. Weil, "On some exponential sums", Proc. Nat. Acad. Sci. 34 (1948), 204-207. Reprinted as pp. 386-389 of his Collected Works I, Springer, New York, 1980.

[Wolfram 1992] S. Wolfram, Mathematica: A System for Doing Mathematics by Computer, 2nd ed., Addison-Wesley, Reading, MA, 1991.

Audrey Terras, Mathematics Department, University of California at San Diego, La Jolla, CA 92093-0112 (aterras@ucsd.edu)

Received May 30, 1995; accepted October 4 